\section{LE PATRIMOINE SAHARIEN TUNISIEN AU DÉFI D'UN TOURISME DURABLE : LE CAS DE TOZEUR ET NEFTA}

\author{
Najem Dhaher \\ Université de Carthage \\ ORCID iD: http://orcid.org/0000-0002-2569-9984 \\ najem_dhaher@yahoo.fr
}

Cómo citar este artículo/Citation: Dhaher, N. (2017). Le patrimoine saharien tunisien au défi d'un tourisme durable : le cas de Tozeur et Nefta. Arbor, 193 (785): a398. doi: http:// dx.doi.org/10.3989/arbor.2017.785n3004

Recibido: 29-11-2016. Aceptado: 08-06-2017.

RÉSUMÉ: Dans les villes sahariennes du sud-ouest Tunisien réputées pour leur culture, leurs traditions et leur patrimoine architectural très particulier, le tourisme a provoqué ces dernières années un effort réflexif autour de la notion de patrimoine destinée à systématiser l'offre touristique et à promouvoir un développement local. Aujourd'hui, le tourisme et le patrimoine dans ces espaces désertiques fragiles et vulnérables sont deux réalités qui marquent profondément les paysages, les activités économiques et culturelles et les mouvements de population. Ces processus, devenus une des préoccupations des acteurs aussi bien publics que privés, ont contribué par les créations nouvelles qu'ils engendrent en termes de représentation et d'utilisation des lieux, à une réinvention de la ville traditionnelle saharienne et de son oasis connus comme biens communs territoriaux par excellence qui nourrissent l'attachement des individus à leurs lieux de vie. En effet, face à un tourisme qui n'est pas toujours inscrit dans des stratégies et des pratiques de développement durable notamment dans ces espaces spécifiques, l'on se demande comment ce dernier peut réduire les nuisances que le tourisme peut occasionner pour le patrimoine dans une perspective de durabilité territoriale?

MOTS CLÉS: Sahara; tourisme; patrimoine; durabilité; développement.

\section{THE TUNISIAN SAHARAN HERITAGE IN THE CHALLENGE OF A SUSTAINABLE TOURISM: THE CASE OF TOZEUR AND NEFTA}

Copyright: @ 2017 CSIC. This is an open-access article distributed under the terms of the Creative Commons Attribution License (CC BY) Spain 3.0.

RESUMEN: En las ciudades saharianas del suroeste de Túnez, afamadas por su cultura, sus tradiciones y por un patrimonio arquitectónico muy particular, el turismo ha provocado estos últimos años un esfuerzo de reflexión en torno a la noción de patrimonio orientada a sistematizar la oferta turística y a promover un desarrollo local. El turismo y el patrimonio en estos espacios desérticos frágiles y vulnerables son hoy dos realidades que marcan profundamente los paisajes, las actividades económicas y culturales y los movimientos de población. Estos procesos, que se han convertido en una de las preocupaciones tanto de los actores públicos como de los privados, han contribuido, a través de nuevas creaciones en términos de representación y de uso de los lugares, a una reinvención de la ciudad tradicional sahariana y de su oasis, conocidos como bienes comunes territoriales por excelencia que alimentan la adhesión de los individuos a los lugares donde viven. Frente a un turismo que no siempre se adhiere a estrategias y a prácticas de desarrollo sostenible especialmente en estos espacios concretos, nos preguntamos precisamente cómo este último puede reducir los perjuicios para el patrimonio que el turismo puede ocasionar en una perspectiva de sostenibilidad territorial.

PALABRAS CLAVE: Sahara; turismo; patrimonio; sostenibilidad; desarrollo. 
ABSTRACT: In the Saharan cities of the Tunisian southwest renowned for their culture, traditions and very specific architectural heritage, tourism in recent years has resulted in a reflexive effort around the notion of heritage intended to systematize the tourist offer and promote local development. Today, tourism and heritage in these fragile and vulnerable desert spaces are two realities which greatly affect the landscapes, economic and cultural activities and movements of the population. These processes, now the concern of public and private actors alike, have contributed, by the new creations which they engender in terms of representation and use of

\section{INTRODUCTION}

Depuis les années 1960, les acteurs de développement touristique en Tunisie n'ont pas pu développer qu'un tourisme balnéaire de masse, avec tous ses impacts négatifs sur l'environnement et la société (Magalie et Houda, 2004). Durant les trois décennies qui suivent les hôtels ont été saupoudrés entre le tissu ancien des villes et leurs plages. En 2007, le nombre d'hôtels touristiques a atteint près de 837 unités. La capacité d'hébergement en lits a été multipliée par 50 entre 1970 et 2010 pour atteindre 250.000 lits et le nombre de nuitées touristiques a été multiplié par plus de 80 , passant de 400.000 à plus de 34 millions. Le nombre de touristes étrangers a atteint en 2014 un peu plus de six millions (Tableau 1).

La plupart du temps, ces équipements ont été réalisés sans aucune vision globale du territoire local, de telle manière qu'ils ont inauguré une grande consommation d'espace, particulièrement agricole. Ce type du produit, qui a montré ses limites aujourd'hui, reste hôtelier et concentré sur le littoral, malgré les potentialités patrimoniales et naturelles recelées par les territoires intérieurs et notamment sahariens.

En fait, le modèle touristique classique (fordiste), fondé sur des pratiques touristiques peu diversifiées et où la dimension relationnelle avec le territoire visité est faible, est en train d'évoluer vers des modèles d'organisation touristique non standardisés et caractérisés par une montée des produits plus humanisés se basant sur le relationnel avec la population locale (Cuvelier, 1998, p. 200). En réalité, suite à la conférence de Rio (1992) et places, to a reinvention of the Saharan traditional city and its oasis known as archetypal territorial joint property, increasing the individuals' attachment to their places of life. Indeed, in the face of a tourism which often does not adhere to strategies and practices of sustainable development, especially in these spaces, we wonder how the latter can reduce the potential damage of tourism on the heritage from a perspective of territorial sustainability.

KEYWORDS: Sahara; tourism; heritage; durability; development. la déclaration de Barcelone (1995), une attention particulière a été accordée au développement touristique par l'instauration des plans de mise à niveau (PMN) dans une logique de " développement durable " qui cherchait à garantir sur le long terme la durabilité de ce secteur dans une perspective de viabilité à long terme (PNUE et OMT, 2006).

Les orientations de l'impulsion de développement touristique par des nouvelles actions, adoptées dans les années 1990-2000, tenant compte de la qualité environnementale et des symboles patrimoniaux des destinations, n'ont pas changé significativement la tendance de développement du secteur en Tunisie faute de prise en compte des critères de durabilité qui respectent les spécificités des territoires. Aujourd'hui, la Tunisie compte près de 250.000 lits hôteliers, dont plus que $90 \%$ concentré sur le littoral.

Le développement touristique dans le Sahara Tunisien a été lancé dans les années 1990 dans le cadre de la stratégie de diversification de l'industrie touristique orientée vers des formes de tourisme plus respectueuses de l'environnement cherchant la valorisation des aménités culturelles et naturelles locales (Écotourisme et tourisme culturel durable en Tunisie). A la fin de l'année 2015, la région Gafsa-Tozeur, incluant Nafta et Tamghza compte 60 hôtels ayant une capacité de 7.289 lits (Office National du Tourisme Tunisien, 2015). La durée moyenne du séjour demeure pourtant faible (1.6 jours). Le taux d'occupation annuelle de cette infrastructure hôtelière est encore modeste (33 \%) avec l'avantage de ne pas se limiter à la saison estivale. En 
Tableau 1. Quelques indicateurs du tourisme Tunisien

\begin{tabular}{|l|l|c|c|c|c|c|c|}
\hline Indicateurs & Unité & 2009 & 2010 & 2011 & 2012 & 2013 & 2014 \\
\hline $\begin{array}{l}\text { Nombre de } \\
\text { touriste }\end{array}$ & Mille & 6901.4 & 6903.4 & 4785.3 & 5950.4 & 6268.9 & 6069.7 \\
\hline $\begin{array}{l}\text { Investissements } \\
\text { touristiques }\end{array}$ & $\begin{array}{l}\text { Million de } \\
\text { dinars }\end{array}$ & 309.3 & 376.4 & 208.2 & 240.1 & 250.1 & 241.9 \\
\hline Emplois directs & Mille & 96 & 96,6 & 96,8 & 96,9 & 96,1 & 96 \\
\hline $\begin{array}{l}\text { Recettes en } \\
\text { devises }\end{array}$ & $\begin{array}{l}\text { Million de } \\
\text { dinars }\end{array}$ & 3471.9 & 3522.5 & 2432.6 & 3175.3 & 3221.4 & 3625.6 \\
\hline
\end{tabular}

Source: Office National du Tourisme Tunisien (ONTT).

effet, la région est plutôt caractérisée par un tourisme de passage dans le cadre de circuits organisés depuis les grandes villes touristiques du littoral. A l'heure actuelle le tourisme au Sahara s'effectue, malgré quelques initiatives citoyennes, principalement sur la base de prestations génériques identiques à celles du modèle balnéaire qui ne peuvent qu'accroitre la pression sur l'environnement et sa durabilité.

Le développement du tourisme saharien a fait l'objet de plusieurs réflexions et débats pour s'adapter rapidement à la multitude des pratiques et aux nouveaux rapports que les touristes entretiennent avec ce territoire de plus en plus intégré dans l'offre touristique tunisienne et de plus en plus visité. La durabilité sociale, éthique et environnementale n'est pas facile à concevoir sur ce territoire fragile (Abichou, Jouve et Sghaier, 2013). Les enjeux de sa mise en tourisme s'avèrent problématiques et considérables.

\section{LE PATRIMOINE SAHARIEN EN TUNISIE, UN ENJEU MAJEUR DE L'ATTRACTIVITE TOURISTIQUE ET DU DE- VELOPPEMENT DES TERRITOIRES}

L'activité touristique tunisienne s'est contentée durant près d'un demi-siècle et jusqu'au début des années 2000 de la monoculture touristique (tourisme hôtelier balnéaire). La conception « muséographique » du patrimoine a prévalu pendant longtemps ce qui a limité les richesses qui ont été sauvegardées et les possibilités de mettre en valeur l'ensemble des spécificités qui fondent les territoires. Depuis les années 1990, l'augmentation de la fréquentation touristique des monuments et des sites historiques sahariens et l'attachement des populations à ces signes de leur identité ont contribué à l'intérêt croissant que leur portent les pouvoirs publics. Le choix d'encourager le développement de ce tourisme n'est pas un choix fortuit ou de circonstance de la part du pouvoir public, mais le résultat d'une réflexion sur le devenir du tourisme tunisien et la stratégie de sa diver- sification dans un environnement compétitif (Ezzedine, 2000 , p. 30). La volonté du gouvernement tunisien était de faire de certains sites sahariens de nouveaux pôles d'attraction touristique. Cette politique s'est surtout affirmée à la fin des années quatre-vingt-dix grâce aux avantages fiscaux accordés aux promoteurs privés. Ainsi, de nombreuses unités hôtelières ont été construites dans les oasis les plus réputées du sud du pays comme celles de Nefta et Tozeur. La sous-occupation hôtelière a amené les pouvoirs publics à construire un aéroport international à Tozeur et à abaisser de $50 \%$ les taxes aéroportuaires pour les compagnies aériennes programmant Tozeur en ligne directe.

Les travaux de restauration et de mise en valeur entrepris dans les médinas et les oasis de Tozeur et Nefta témoignent du nouveau potentiel de croissance que représente le patrimoine pour le développement local. Ce patrimoine désertique qui représente une composante essentielle de la vie et de l'économie locale est souvent marqué par une implication citoyenne sur le terrain (Magalie et Houda, 2004). La participation du tourisme à la révélation des ressources patrimoniales locales a débouché sur leur valorisation directe par des pratiques, des produits et des services porteurs de représentations de la destination et déclencheurs d'un certain développement (El Gaied et Meyer, 2014).

Le regain d'intérêt pour le patrimoine saharien et l'investissement public et privé dans les centres historiques sahariens pour des projets touristiques à valeur culturelle et patrimoniale reste encore timide et hésitant en Tunisie. Souvent considéré comme patrimoine d'héritage au sens muséographique du terme, il tend aujourd'hui à prendre un caractère marchand, en tant que produit à valoriser économiquement à travers le tourisme ${ }^{1}$. Ceci a été encouragé surtout par l'importance de l'attraction des sites historiques et des manifestations culturelles auprès des touristes. 
Tableau 2. Quelques indicateurs du tourisme saharien à (Kebili, Tozeur, Gafsa) en 2010

\begin{tabular}{|l|c|c|c|c|}
\hline & Kébili & Tozeur & Gafsa & Tunisie \\
\hline Arrivées & 370428 & 300852 & 59330 & 6903400 \\
\hline Nuitées globales & 401895 & 505954 & 94484 & 36200000 \\
\hline Taux d'occupation (en\%) & 26 & 29,4 & 26,6 & 50,3 \\
\hline Durée de séjour (en jours) & 1,1 & 1,7 & 1,6 & 5,2 \\
\hline Emplois & 1504 & 1963 & 540 & 96900 \\
\hline Nombre de lits & 4306 & 6362 & 1215 & 241500 \\
\hline Recettes (en 000 dinars) & 44308 & 55655 & 10393 & 3522500 \\
\hline
\end{tabular}

Source: Office du Développment du Sud, 2012.

La question patrimoniale s'invite fréquemment au cœur de plusieurs projets urbains et territoriaux pour une mise en relation du patrimoine et du tourisme ${ }^{2}$. Cet intérêt a été provoqué en réalité juste après l'inscription de la ville saharienne ancienne de Tozeur par I'UNESCO sur la Liste du Patrimoine Mondial de l'Humanité en 1988.

Dans le contexte actuel de la concurrence touristique, le patrimoine culturel saharien est devenu un enjeu important de l'attractivité touristique et du développement des territoires. Toutefois, la relation s'avère prégnante et parfois conflictuelle du tourisme au patrimoine et à la culture au Sahara. Elle devient problématique lorsque l'on évoque la question de la durabilité. Le processus d'empowerment ou encore la place des populations locales dans cette action et leur participation dans la patrimonialisation se présente à son tour comme un enjeu majeur lié non seulement aux identités culturelles de la population concernée, mais aussi aux nouvelles perspectives de cette patrimonialisation et aux enjeux d'un développement touristique saharien durable.

Certaines villes et oasis historiques sahariennes connaissent depuis les années 1990 cette situation à travers des rapports complexes entre préservation du patrimoine et sa mise en tourisme. La politique de réhabilitation et de valorisation du patrimoine culturel entreprise par les différents acteurs a révélé de nouveaux enjeux liés aux outils et méthodes utilisés, à la gestion des incidences occasionnées par le processus de mise en tourisme et aux techniques qui concernent les savoir-faire ${ }^{3}$.

\subsection{Valorisation du patrimoine par le tourisme : une dynamique confrontée à la logique marchande}

Aujourd'hui, le tourisme a provoqué dans beaucoup de villes sahariennes tunisiennes un effort réflexif autour de la notion de patrimoine destinée à systématiser l'offre touristique et à promouvoir un développement économique et culturel local. Suite à cette prise de conscience des valeurs techniques, culturelles et sociales que le patrimoine recèle, son intégration dans les contextes urbains, économiques et sociaux contemporains est devenue un enjeu pour l'aménagement et l'identité de la ville. Toutefois, le tourisme peut-il revaloriser ces espaces sans pour autant compromettre leur existence et leur durabilité ? Car, on peut craindre que le tourisme qui semblait être dans ces espaces un vecteur d'une prise de conscience de la valeur du patrimoine comme élément fondateur de l'identité culturelle saharienne locale, ne soit plus qu'une forme de " consommation culturelle » et n'aboutisse au contraire à une banalisation rapide du patrimoine qui pourrait porter atteinte à l'urbanisation saharienne historique et conduire à la perte de l'identité et aux paysages patrimoniaux locaux.

Tableau 3. Activités traditionnelles et artisanales liées au tourisme

\begin{tabular}{|l|l|l|l|}
\hline & Tozeur & Kebili & Gafsa \\
\hline $\begin{array}{l}\text { Principales } \\
\text { activités }\end{array}$ & Broderie, brique, tissage & $\begin{array}{l}\text { Maroquinerie, broderie, tissage, } \\
\text { habit traditionnel }\end{array}$ & Broderie, tissage \\
\hline $\begin{array}{l}\text { Matières } \\
\text { premières }\end{array}$ & $\begin{array}{l}\text { Alfa, laine, feuille et bois de palmier, } \\
\text { argile }\end{array}$ & Fibre, laine & Argile, fibres, laine \\
\hline
\end{tabular}

Source: Office National de l'Artisanat Tunisien (ONAT), 2012. 
Les villes sahariennes de Tozeur ${ }^{4}$ et Nefta ${ }^{5}$ avec leurs centres historiques empreints d'une forte tradition culturelle sont aussi définies par leur situation entre deux grandes entités paysagères: l'oasis et l'espace désertique (figure 1). Cela a constitué, pour les entités qui ont conservé remarquablement leur trame urbaine intra-muros mais également pour leurs oasis traditionnelles, un atout pour le développement du projet de leur valorisation et la création de nouveaux produits touristiques susceptibles de contribuer à la compétitivité de ces territoires plutôt marginalisés.

Les festivals culturels dans ces deux villes offrent à leur tour un champ d'observation très propice à l'étude des processus de sauvegarde et de construction de la valeur patrimoniale immatérielle des populations du désert tunisien, surtout du point de vue communautaire. Ces manifestations qui se déroulent chaque année au démarrage de la saison touristique d'hiver présentent de divers tableaux de la vie des populations sahariennes, des savoir-faire artisanaux et artistiques qui attirent surtout des touristes maghrébins et européens. Ces expressions culturelles semblent jouer un rôle de plus en plus important non seulement dans la sauvegarde des traditions culturelles sahariennes mais aussi dans l'économie locale qui mise sur son patrimoine immatériel à travers l'implication de la population détentrice de ce dernier. Souvent, pendant ces festivals, l'afflux de visiteurs dépasse la capacité d'accueil des hôtels et des maisons d'hôte. Cette dynamique encourage parfois certaines familles de la région à prêter leurs maisons et locaux aux visiteurs d'après un commerçant de Tozeur (Babnet Tunisie).

Figure 1. L'oasis de Nefta : une palmeraie nichée au fond d'une dépression

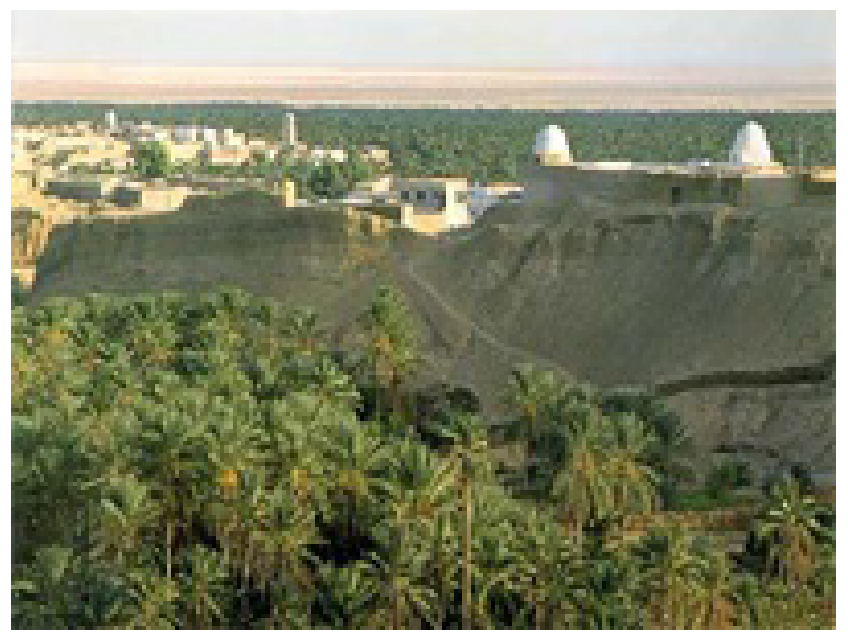

Figure 2. L'architecture traditionnelle à Tozeur : un atout pour le développement touristique
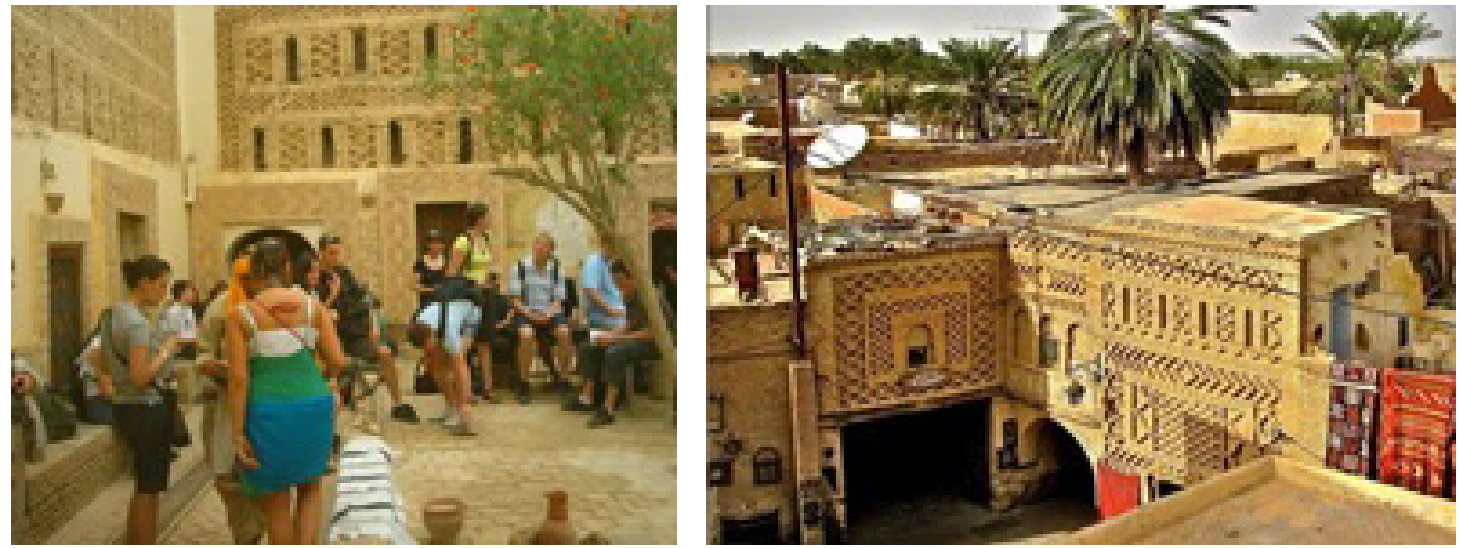

Source: Commune Tozeur Nefta 
Mais, I'institutionnalisation de cet héritage dans la confrontation des logiques des différents acteurs concernés s'avère problématique. En effet, entre un désir d'appropriation qui conforte le sentiment d'appartenance et d'identification à la localité et un désir de marchandisation et d'instrumentalisation pour attirer les touristes avec l'appui d'instruments et d'activités qui ne sont pas toujours l'expression de la culture immatérielle ${ }^{6}$, le patrimoine se trouve ainsi pris dans une tension (Dhaher, 2012a).-

En effet si les commerçants comme le propriétaire d'un local d'artisanat au souk Errobô à Tozeur, soulignent les bienfaits de la commercialisation du tourisme saharien sur l'activité économique dans la région, les associations de sauvegarde du patrimoine et l'élite locale dénoncent certaines opérations de réhabilitation et d'implantation d'équipements d'accueil mal adaptés et dénués de toute référence culturelle visant tout simplement les bénéfices économiques qui ont abouti, disent-ils, à la banalisation de plusieurs sites traditionnels. Le rôle des populations locales dans la mise en tourisme du patrimoine alimente les débats depuis la révolution de 2011. Les communautés locales réclament une participation plus effective dans la programmation et l'aménagement des projets de tourisme qui permettra une redistribution équitable des recettes. Un certain " pro-poor tourism » ou " tourisme pro-pauvre ${ }^{7}$ » est réclamé aujourd'hui par une grande partie de la population saharienne.

Les tentatives mises en œuvre pour faire du patrimoine un élément moteur du développement local paraissent justifiées surtout dans des zones sahariennes en difficulté et avec une activité touristique en pleine expansion. Les opérations de préservation et de réhabilitation des quartiers traditionnels de Tozeur et de Nefta ont constitué une action salvatrice importante non seulement pour la sauvegarde du patrimoine historique qui est en péril mais aussi pour son intégration dans le processus du développement de la ville voire de la région. Cependant, il paraît difficile pour les responsables locaux de faire converger préoccupations patrimoniales, touristiques et objectifs du développement durable.

\section{PATRIMONIALISATION ET ENJEUX D'UN DEVELOP- PEMENT TOURISTIQUE SAHARIEN DURABLE}

Certes, la protection du patrimoine, sa survie et sa transmission à des générations futures dépendent à notre sens pour beaucoup de son appropriation, de sa fonctionnalité et de son rôle dans la société actuelle. Cependant à travers la recherche " coute que coute » des aménités du territoire saharien en vue de leur mise en scène et de leur mise en vente, des conflits émergent (Dhaher, 2016). Aujourd'hui, un fort risque de folklorisation des cultures locales apparait dans des représentations marchandes souvent dévoyées malgré la présence de textes juridique ${ }^{8}$ qui obligent tout promoteur touristique à établir une étude d'impact sur l'environnement préalablement à toute réalisation. La petite agriculture familiale traditionnelle a été profondément marginalisée à la suite de la spéculation foncière liée à la mise en tourisme de l'oasis ancienne, de la chute de la main d'œuvre et de la pression sur les ressources en eau.

Les extensions illicites des périmètres irrigués aux dépens des oasis traditionnelles ont été encouragées par l'accès incontrôlable à l'eau et au foncier et par une urbanisation anarchique et galopante aux alentours et à l'intérieur des oasis. Le développement d'un tourisme fortement consommateur d'eau (zones hôtelières de Douz, Nefta ou Tozeur par exemple) avec des équipements pas toujours adaptés à l'environnement (piscines, golfs) a accéléré ce processus et a aggravé dans l'ensemble des régions oasiennes, la raréfaction des ressources en eau, la dégradation des sols et a accéléré l'abandon de l'activité agricole et l'atteinte à la biodiversité (figure 3). En février 2012, le déficit hydrique de la zone est tel que le débit des pompages ne dépasse pas les 1.000 litres par seconde. Certaines études montrent une baisse de 0,5 à 2 mètres du niveau piézométrique et le tarissement de 194 sources de la région. Selon ces études,

Tableau 4. Ressources en eau et exploitation dans les oasis et villes de Tozeur, Kébili et Gafsa

\begin{tabular}{|l|c|c|c|c|c|}
\hline & Ressources en eau (Mm3/an) & $\mathbf{1 9 8 5}$ & $\mathbf{1 9 9 5}$ & $\mathbf{2 0 0 5}$ & $\mathbf{2 0 1 0}$ \\
\hline Tozeur & 33,6 & 17,9 & 27 & 32,31 & 38,58 \\
\hline Kébili & 5,5 & 0,7 & 1,4 & 0,28 & 1,5 \\
\hline Gafsa & 33,3 & 14 & 37,6 & 34,3 & 42,3 \\
\hline
\end{tabular}

Source: Direction Générale des Ressources en Eau (DGRE), Tunis. 
un touriste consomme en moyenne 560 litres d'eau par jour, contre 100 litres pour un habitant (Stratégie de Développement Durable des Oasis en Tunisie, p. 99). Tout cela conduit au besoin de mettre au premier plan d'intérêt pour le développement durable des oasis la gouvernance des ressources naturelles dans les oasis en Tunisie (Sghaïer, 2010).

Dans la région sud-ouest du Sahara Tunisien, et suite à une " touristification " inadaptée et incontrôlée et une surexploitation des nappes, l'irrigation s'appuie essentiellement sur le pompage de l'eau des aquifères fossiles non-renouvelables, avec une diminution dans la qualité et la quantité des ressources en eaux.

Bref, la volonté des acteurs locaux d'articuler le patrimoine au développement notamment économique dans le Sahara nécessite une gestion qui permet de respecter les intérêts des communautés locales impliquées dans le développement du tourisme et d'assurer une exploitation durable du patrimoine. Les acteurs publics et privés doivent comprendre les enjeux inhérents au fait de mettre en tourisme un site saharien fragile ou un quartier historique fréquenté par les touristes et de garantir sa survie, sa pérennisation et sa transmission à des générations futures. Les quartiers historiques, le patrimoine immatériel et le paysage oasien sont avant tout une richesse patrimoniale qui contribue à l'attractivité des territoires, dont ils sont des éléments identitaires forts.

La mise en tourisme du patrimoine à Tozeur a généré relativement de la croissance et des emplois en majorité temporaires. L'industrie touristique a eu un effet de fixation de certains traits de la culture locale traditionnelle (Puig, 2004), mais le constat n'est pas sans poser de problèmes.
D’ailleurs, sous le prétexte de faciliter l'accueil d'un nombre croissant de touristes, la réalisation des opérations de réhabilitation et les implantations des équipements d'accueil mal adaptés et dénués de toute référence culturelle visant tout simplement les bénéfices économiques ont abouti à la banalisation de plusieurs édifices traditionnels et ont conduit à la perte progressive de leur identité culturelle. Les " houchs» traditionnels donnant sur les axes principaux ont été transformés en boutiques. Souvent ces transformations ont été effectuées sans tenir compte de l'identité architecturale du tissu traditionnel (réduction des patios pour avoir plus d'espace, appropriation de la galerie, etc.). La concentration des équipements au centre des quartiers historiques a été défavorable à l'écoulement des flux véhiculaires qui augmentent considérablement (figure 4).

Dans les quartiers historiques, la circulation piétonnière intense générée par la concentration des activités de commerce et de service est également devenue très difficile surtout avec l'occupation des trottoirs pour les stationnements et les activités informelles. Les espaces domestiques, quant à eux, ont été soumis à de nombreuses évolutions qui témoignent de la diffusion de nouvelles valeurs urbaines.

Certaines manœuvres des acteurs locaux qui ne s'accordent pas avec l'identité locale tout en tentant de répondre aux représentations touristiques régentent parfois vers une folklorisation du patrimoine, soit dans l'application de matériaux qui ne correspondent pas au statut de l'édifice, soit en modifiant les structures de base et en corrigeant imparfaitement les défectuosités de l'architecture originelle, soit aussi en donnant à certains ensembles patrimoniaux une fonction inconvenable.

Figure 3. Le tourisme au Sahara : des équipements fortement consommateurs d'eau
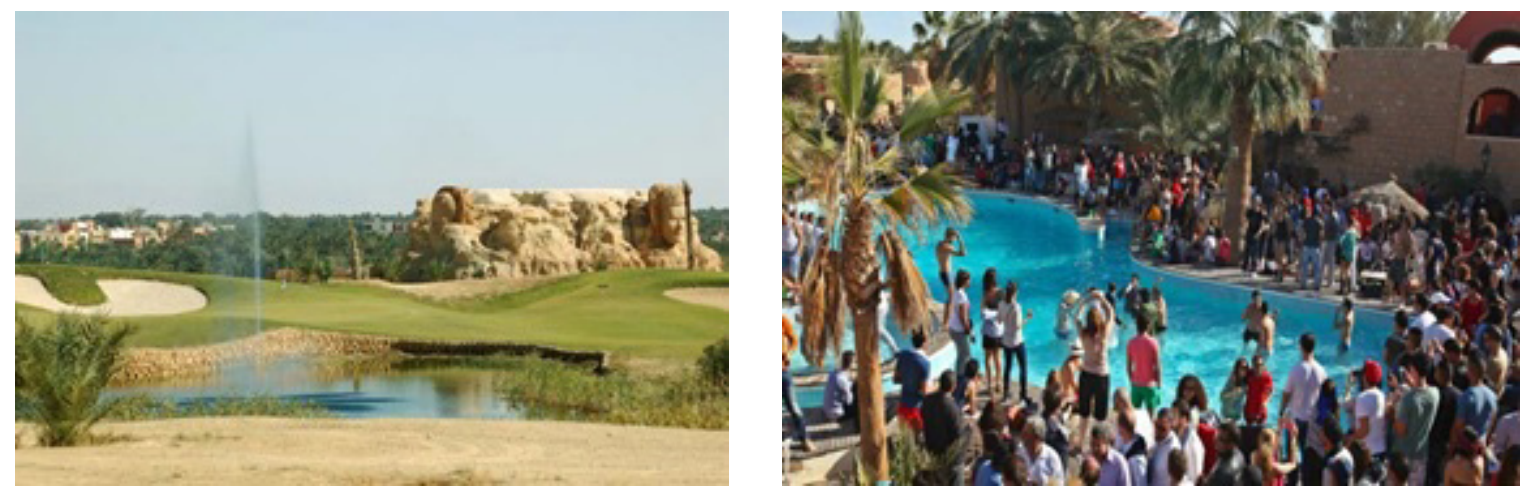

Source: Commune Tozeur, 2010. 
Figure 4. Espaces traditionnels et fonctions contemporaines dans la ville ancienne de Tozeur

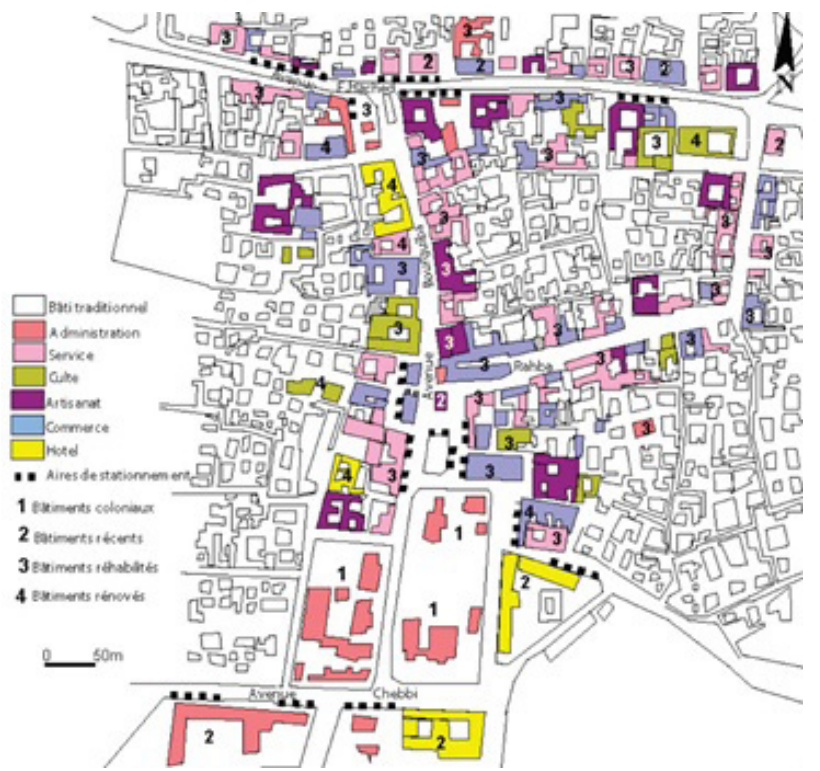

Source: Dhaher, 2012b.

La petite agriculture familiale traditionnelle saharienne s'est ainsi retrouvée exposée non seulement à une agriculture exportatrice mais surtout à la concurrence d'un tourisme qui capte tous les investissements (Carpenter et Gana, 2012). Certains agriculteurs ont transformé leurs oasis afin de profiter de l'aubaine de l'essor du tourisme. Aujourd'hui, le territoire oasien n'est plus considéré comme nourricier, mais comme espace de loisir et de "marketing ». Le paysage référent est celui de l'oasis traditionnelle qui est entretenue et recréée dans une logique de jardinage où la dimension esthétique prime (Carpenter et Gana, 2012). Cette réalité commence à entraîner un bouleversement des hiérarchies économiques et sociales, en détournant la main d'œuvre vers les services touristiques au détriment des activités traditionnelles nécessaires à l'entretien du milieu oasien.

Le passage d'une logique de préservation de ces sites à une action valorisante durable intégrant les territoires sahariens nécessite l'implication et l'engagement de tous les acteurs concernés pour concilier protection du site et développement culturel touristique en vue de redynamiser ces territoires en déclin et apporter l'onction d'une valeur universelle à tout ce qu'un territoire contient. Les acteurs locaux semblent singulièrement en panne d'idées, de financement et en conséquence d'outils, pour lire le phénomène et y répondre par un accompagnement approprié. Selon les propos de Furt et Michel (2006), une mise en tou- risme de l'identité culturelle et surtout sa valorisation touristique doivent être respectueuses de cette même identité et lui garder son authenticité : « le tourisme est comme le feu, il peut cuire ta soupe ou brûler ta maison » (Furt et Michel, 2006).

Si le volet économique du développement durable a été assez reconnu, les volets social et environnemental traînent encore à se faire reconnaître. Le changement des traditions locales et des modes de vie a induit un déclin de ces espaces. La fonction d'origine a été perdue et de nouvelles fonctions notamment touristiques sont en train d'accaparer certains espaces. La forme urbaine dense et compacte des quartiers traditionnels, qui crée de l'ombre et minimise les surfaces extérieures exposées au rayonnement solaire, connait sous l'effet du tourisme de mauvaises interventions souvent insensibles aux valeurs historiques du bâti (figure 5). La composition architecturale horizontale et introvertie des médinas qui permet de mieux s'adapter aux conditions climatiques du milieu connait quelques extensions en étages qui constituent un choc visuel, contradictoire avec la morphologie traditionnelle. Les structures et les textures qui empêchent l'expansion du désert et les effets des tempêtes de sable, tout en créant des espaces ombragés et qui ont été modifiées suite à l'effet du temps et à l'abandon font l'objet, tout de même, d'une valorisation plutôt réfléchie par les associations de sauvegarde des médinas. L'utilisation de la brique donne de plus en plus 
sa caractéristique à l'architecture des villes de Tozeur et Nefta. Si l'existence d'importants gisements d'argile fut une raison du choix des maçons, il en est d'autres qui tiennent à la qualité propre de la brique. Sa porosité lui permet d'absorber facilement les eaux de pluie et de conserver une relative fraîcheur.

Figure 5. Certaines opérations d'extension ou de restauration sont en déphasage avec la mémoire des lieux

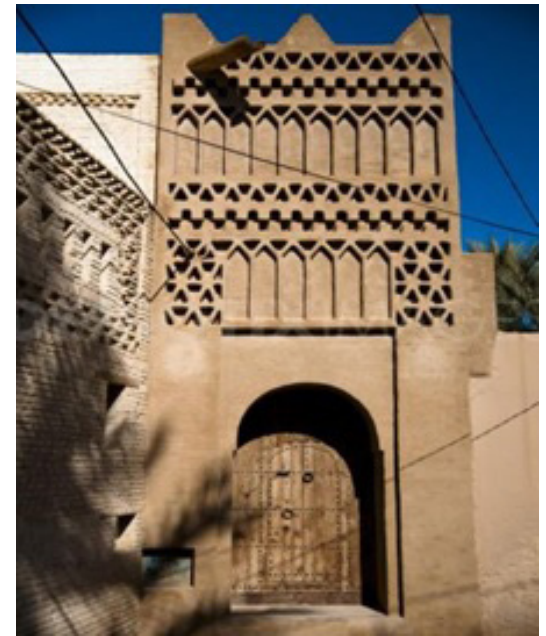

Source: Commune Tozeur.

Certes, la protection du patrimoine et sa transmission à des générations futures dépendent à notre sens pour beaucoup de son occupation, de sa fonctionnalité et de son rôle dans la société actuelle. Mais, si les espaces patrimoniaux ne seront pas convenablement adaptés aux conditions d'habitabilité et de fonctionnement actuel, ils entrainent des dysfonctionnements dans les pratiques de l'espace dus à des temporalités et à des spatialités des touristes qui se superposent à celles des habitants permanents (Neyret et Colin, 2003). Pour qu'il y ait patrimonialisation, " il faut également que l'objet patrimonial puisse acquérir une valeur économique " (Garat, Gravari-Barbas et Veschambre, 2008, p. 2). Autrement dit, il faut qu'à l'intérêt esthétique, historique s'ajoute une valeur d'échange. Cette gestion du patrimoine s'avère encore délicate en Tunisie surtout quand il s'agit de concilier la rentabilité économique avec la préservation des sites et des monuments et leur durabilité. Le tourisme pris en compte dans les villes de Tozeur et Nefta comme un des moyens de valorisation du patrimoine n'a pas réussi à être son rédempteur même si certaines initiatives entreprises en matière de développement local d'un tourisme qui se veut durable veulent le croire. L'exemple remarquable du projet en cours de réalisation entre l'association de sauvegarde de la médina de Tozeur et la coopération allemande ${ }^{9}$ qui vise à assurer un processus participatif de tous les acteurs et à accompagner un développement touristique durable à travers une valorisation des potentialités culturelles et naturelles a eu un effet d'entraînement remarquable.

La population, bien qu'encore très mal impliquée, est de plus en plus sensible aux valeurs patrimoniales et à leur protection comme le montrent certaines actions de rénovation. Mais la réhabilitation du patrimoine n'est pas toujours comprise et acceptée par les responsables locaux et les concitoyens ce qui nécessite une prise en compte de l'identité culturelle et des besoins des habitants et mettre en œuvre des dispositifs sociaux d'accompagnement. C'est là l'objectif primordial de la patrimonialisation urbaine durable. D'ailleurs, la tenace réalité du rapport souvent inégal entre les touristes et les populations locales et le déséquilibre entre ces derniers et les promoteurs touristiques invitent à penser de nouvelles formes de régulation (Collectif, 2006). II s'agit de coordonner la sauvegarde du patrimoine à

Figure 6. Aménagement de la corbeille de l'oasis de Nefta
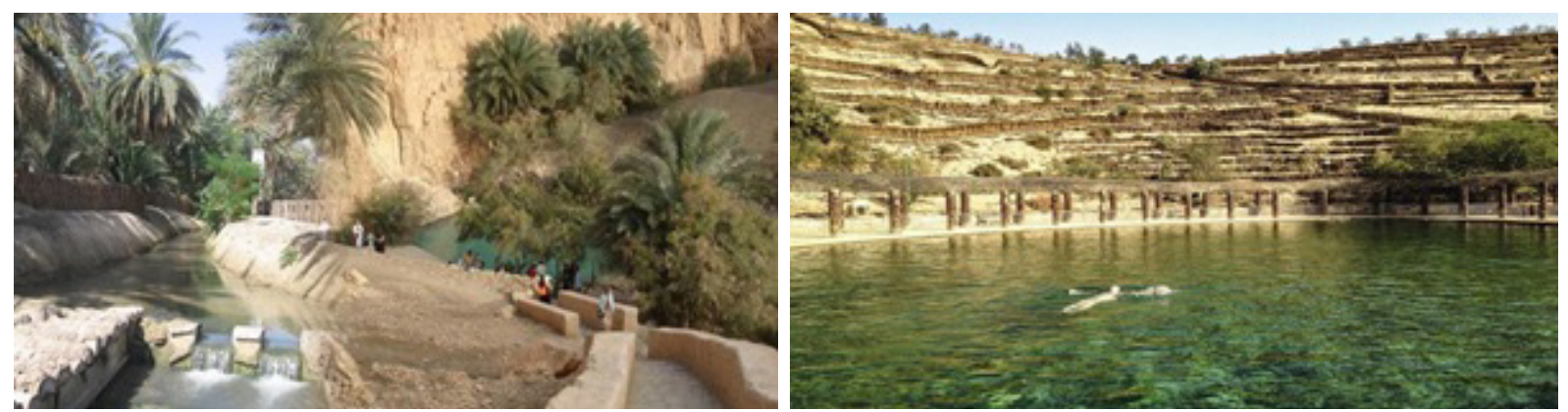

Source: Commune Nefta. 
d'autres mesures telle la requalification durable de l'espace public en faveur des usagers de la ville, la gestion de la mobilité urbaine, l'esthétique urbaine et autres besoins des habitants.

Les modalités d'un développement durable de ces espaces uniques et fragiles, afin de préserver le patrimoine de la ville aussi bien pour les générations présentes que futures, nécessitent l'amélioration des conditions de vie de la population locale qui doit être actrice réelle de son développement et tirer les bénéfices de l'activité touristique. "Le développement du tourisme saharien durable doit s'inscrire dans une dynamique qui articule des modes de production et de consommation responsables, tout en offrant aux populations qui vivent, travaillent ou séjournent sur ces espaces des avantages socio-économiques équitablement répartis ${ }^{10}$.

Occupés par une population locale en difficulté économique, le développement dans des espaces sahariens contraignants et précaires représente aujourd'hui une problématique à part entière. En effet, malgré un potentiel en termes d'aménités naturelles et culturelles, l'environnement humain et les écosystèmes oasiens sont menacés par un ensemble de facteurs liés à la modernisation, à la modification des pratiques de gestion traditionnelles et essentiellement à une urbanisation croissante et souvent incontrôlée. Toutefois, l'adoption des valeurs du tourisme durable ne doit-elle pas à la fois réduire les disparités sociales et privilégier une répartition spatiale équitable notamment dans un pays en voie de développement comme la Tunisie ? Les initiatives en matière de tourisme responsable, solidaire, durable ou éthique conformément à la Charte du tourisme durable (Conférence mondiale du Tourisme durable, à Lanzarote, Iles Canaries, Espagne, les 27 et 28 avril 1995), veulent le croire, en transformant le loisir en un échange équitable et un instrument d'écodéveloppement. Mais la situation en Tunisie reste mitigée et hésitante malgré quelques initiatives citoyennes (voir 2.1) qui croient encore en un tourisme durable permettant le développement et respectant aussi bien les communautés autochtones que leurs patrimoines naturels et culturels.

\section{EMERGENCE DE NOUVELLES PRATIQUES DE SAUVE- GARDE ET DE PROMOTION DU PATRIMOINE SAHARIEN}

A vrai dire, les touristes sont de plus en plus nombreux à prendre en compte les critères de durabilité dans leurs choix d'hébergement et de transport. La quête d'authenticité selon plusieurs recherches est devenue l'un des premiers motifs de déplacement chez les touristes.

Tableau 5. Principaux motifs de déplacement de touristes au Sahara Tunisien

\begin{tabular}{|l|c|}
\hline Circuits au Sahara & $35 \%$ \\
\hline Oasis et Sahara & $31 \%$ \\
\hline Hôtels et villages de vacances & $17 \%$ \\
\hline Ksars et villages berbères & $13 \%$ \\
\hline Animations hôtelières & $2 \%$ \\
\hline
\end{tabular}

Source: Divers agences de voyage, Office National du Tourisme Tunisien, Souissi (2011).

Du coup, la valorisation par le tourisme des aménités patrimoniales et environnementales dans des espaces sahariens fragiles et vulnérables amène à repenser les considérations environnementales et humaines de durabilité qui sont aussi importantes que les variables de rentabilité et qui devraient être au centre de toute réflexion sur le développement touristique territorial.

Aujourd'hui, après l'échec du modèle balnéaire et face à un processus de mise en tourisme du patrimoine qui est en train de conduire à une certaine marchandisation des territoires, de nouvelles pratiques plutôt citoyennes émergent ainsi qu'une prise d'initiatives par certaines associations pour concevoir et réaliser des actions concrètes qui essayent de réconcilier tourisme et développement durable et d'inventer un " tourisme citoyen ». Si à priori, faire évoluer le tourisme et introduire les logiques de durabilité est incompatible avec le souci des héritages urbains sahariens, on pourra s'interroger à quelles conditions la mise en tourisme du patrimoine saharien peut-il s'engager dans la durabilité et l'équité ?

La révolution de 2011 qui a bien révélé les défaillances du modèle touristique balnéaire, a encouragé des habitants de Tozeur et Nefta à se mobiliser pour faire renaître un tourisme saharien traditionnel des années 1960 basé sur la diversité des paysages, des cultures et des habitats traditionnels. Beaucoup d'habitants sont unanimes devant la fragilité de l'équilibre entre la vocation résidentielle et la multifonctionnalité de leurs quartiers, entre la vocation agricole et l'usage de leurs oasis et devant une industrie touristique de plus en plus dominante. Aujourd'hui, on assiste à l'émergence de nouvelles pratiques basées sur des formes de valorisation patrimoniales des ressources sahariennes et oasiennes marquées par l'orientation de la demande vers 
une consommation plus " authentique " et porteuse de plus de « sens ». L'héritage culturel local dans ces territoires se veut être un objet quotidien et non un objet de contemplation, exposé dans des musées et transformé souvent en folklore. C'est dans ce cadre que survient le réaménagement du circuit touristique écologique "Râs el Aïn " de Nefta ${ }^{11}$ ou le développement de l'agro-tourisme $^{12}$ dans les oasis de Tozeur à travers l'aménagement de fermes modèles illustrant l'ancienne agriculture traditionnelle. La protection et la valorisation des rares oasis anciennes qui gardent encore leur système d'irrigation traditionnel ainsi que les tentatives lancées par les autorités locales pour mettre en valeur les vieilles palmeraies répondent aussi aux attentes des flux touristiques.

La restauration "d'une architecture sans architectes » (Daghari, 2002) tente d'allier, dans le même geste, le matériau de construction et le savoir-faire de I'artisan-maçon. La brique traditionnelle de Tozeur qui symbolise le caractère particulier de l'architecture locale a fait l'objet d'un décret municipal ${ }^{13}$ pour retrouver sa place comme l'un des piliers de l'économie de la ville ${ }^{14}$. De là, le tourisme dans la région commence à se présenter comme un atout pour la conservation du patrimoine et sa durabilité.

Ce nouveau rôle du patrimoine a permis d'offrir quelques bénéfices socio-économiques sur l'environnement du site, par la création de petits commerces qui ont permis de créer des emplois permanents sur le site.

Tableau 6. Indicateurs sur les actifs artisans

\begin{tabular}{|l|c|c|c|}
\hline & Tozeur & Kébili & Gafsa \\
\hline Artisans inscrits & 5122 & 2570 & 7672 \\
\hline Entreprises artisanales & 34 & 124 & 122 \\
\hline
\end{tabular}

Source: Office National de l'Artisanat Tunisien (ONAT), 2013.
Grace à l'implication citoyenne et associative qui est en train de promouvoir des formes de tourisme plus douces, certains sites sont non seulement préservés et réhabilités mais également enrichis par de nouvelles mobilités touristiques, commerciales et de loisir. Certaines aménités culturelles et environnementales dans cette région sont souvent les supports d'un processus de transmission générationnelle de pratiques sociales et de savoir-faire locaux. Malgré ces dynamiques territoriales locales qui essayent de promouvoir une activité touristique appropriée respectueuse de l'écosystème et génératrice de revenus localisés, le tourisme saharien et oasien ne semble pas à ce jour être en mesure d'assurer la durabilité de ces écosystèmes devant l'absence d'une stratégie publique en faveur de ces nouvelles dynamiques touristiques.

\section{CONCLUSION}

L'élargissement de la notion de tourisme saharien depuis la fin des années 1980 jusqu'à nos jours et le sens nouveau accordé à son utilité prennent une nouvelle signification. Aujourd'hui, ce tourisme est intégré dans une diversité d'objets mobilisés par différents outils pour développer l'offre touristique surtout après l'échec du tourisme balnéaire. En effet, la littérature internationale souligne qu'à un niveau global on passerait d'un tourisme " de masse ", standardisé, à des formes de tourisme alternatives, marquées par l'orientation de la demande vers une consommation plus « authentique » et porteuse de plus de " sens ». Au Sahara, ces formes alternatives de tourisme ne peuvent être, par essence, que limitées, puisque les actifs spécifiques sur lesquels elles reposent peuvent être assez vite mis en danger aussi bien par l'instrumentalisation des aménités culturelles et naturelles saharienne, devenu un produit marchand de grande consommation que par une

Figure 7. Production et utilisation de la brique traditionnelle de Tozeur
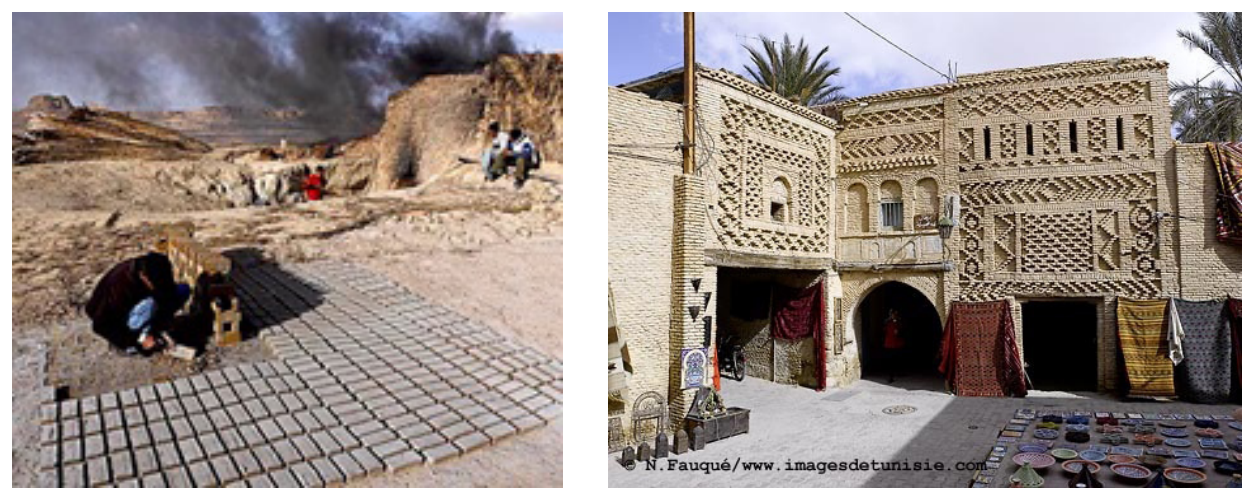

Source: www.tunisiadailyphoto.com 
augmentation des pratiques touristiques au-delà de la capacité de charge liée à ces territoires (Minvielle, Smida et Majdoub, 2007).

La Déclaration de Budapest, en 2002, énonce la nécessité de « maintenir un juste équilibre entre la conservation, la durabilité et le développement, de façon à protéger les biens du patrimoine mondial grâce à des activités adaptées contribuant au développement social et économique et à la qualité de vie de nos communautés ". En 2012, le quarantième anniversaire de la Convention de 1972 a permis la construction d'une synthèse entre les deux objectifs du développement et de la conservation.

L'évolution qu'a connue le tourisme au Sahara Tunisien depuis des décennies, conduit à se poser tout d'abord la question de la spécificité du tourisme dans ces espaces. Ce tourisme, perçu comme élément perturbant à maîtriser (Bourdeau, Gravari-Barbas et Robinson, 2012), constitue un des enjeux entre conservation du patrimoine et développement des territoires.

De ce point de vue se pose alors la question de savoir par quelles adaptations les cultures et les identités, qui représentent le véritable patrimoine humain saharien, peuvent y survivre sans tomber dans les travers de l'acculturation, de l'assujettissement ou de la folklorisation. L'enjeu de développement durable est affirmé au même titre que celui de la conservation, marquant bien ce nouveau paradigme de l'association tourisme-patrimoine, à travers la promotion d'un tourisme durable (Gravari-Barbas et Jacquot, 2013). Les documents préparatoires à la résolution adoptée à Brasilia puis à Saint Pétersbourg ${ }^{15}$ précisent le contenu de ce nouveau programme.

La relation multiple, prégnante et parfois conflictuelle du tourisme au patrimoine et à la culture notamment dans des espaces marginalisés et vulnérables renvoie souvent à la notion de "Tourisme durable ", et se réfère à la mise en tourisme des héritages culturels. Ces éléments constituent des " aménités patrimoniales » qui viennent s'articuler aux aménités environnementales pour constituer la base de l'attraction touristique. Le tourisme saharien Tunisien s'y intéresse donc, mais souvent de ma- nière différente suivant son modus operandi malgré quelques efforts des acteurs publics qui œuvrent pour préserver la soutenabilité de l'attraction de leur territoire. Toutefois, les politiques de développement touristique sont jusqu'à maintenant centralisées, où le rôle des acteurs territoriaux locaux n'est pas confirmé. Ceci a handicapé le développement du tourisme dans des espaces sahariens fragiles et marginalisés qui devra être appréhendé dans et par le territoire, comme espace de vie et d'action, mettant au centre de ses préoccupations la contrainte patrimoniale autant que la fragilité et la vulnérabilité environnementales.

Certes, l'intérêt pour le patrimoine saharien et sa valorisation touristique en vue d'accroître sa rentabilité économique sont légitimes. Toutefois, certaines actions de "touristification " du patrimoine, bien que relativement bénéfiques pour l'économie locale, risquent de ne pas participer à un développement durable surtout lorsque le tourisme culturel ne s'inscrit pas clairement dans des stratégies et des pratiques de développement durable. Aujourd'hui, on peut craindre que le tourisme qui semblait être le vecteur d'une prise de conscience de la valeur du patrimoine comme élément fondateur de l'identité culturelle locale, ne soit plus qu'une forme de " consommation culturelle " et n'aboutisse au contraire à une banalisation rapide du patrimoine saharien. Les rares actions citoyennes de valorisation par le tourisme de I'héritage culturel saharien sont encore en germe et ont besoin de soutien, d'accompagnement et d'encouragement. Le besoin de l'empowerment ou du renforcement des capacités par la formation et le financement s'avèrent nécessaires, à travers une stratégie de tourisme durable où le spatial autant que l'humain, l'économique et l'environnemental doivent être pris en considération (Boukherouk, 2012). Cette question est en effet encore émergente en Tunisie, et l'articulation des actions locales et des politiques publiques reste problématique. Et cela met en cause l'une des responsabilités majeures des différents acteurs territoriaux qui réside dans l'obligation morale de léguer aux générations futures le patrimoine que nous-mêmes avons hérité. 


\section{NOTES}

1. Les investissements dans ces zones bénéficient des avantages prévus par les articles 23, 24, 25 et 26 du code d'incitation aux investissements.

2. Le décret $n^{\circ}$ 94-426 du 14 février 1994 délimite les zones d'encouragement au développement régional pour la promotion du tourisme saharien.

3. Une formation de techniciens et d'artisans dans le savoir-faire traditionnel de la construction et de la production de matériaux a été dispensée par des maîtres artisans traditionnels dans le cadre d'un projet d'étude, de sauvegarde et de valorisation des villes-oasis financé par la région italienne de Reggio Calabre.

4. Tozeur est réputée pour son oasis et l'abondance de sa végétation étagée et luxuriante. Le vieux quartier des Ouled El-Hadef, tout comme le centre moderne, présentent des façades au décor géométrique, fait de briques traditionnelles de couleur ocre.

5. Nefta est connue par la palmeraie la plus vaste de Tunisie. La ville a une réputation religieuse, avec ses nom- breuses mosquées et marabouts, dont certaines datent du $16^{\text {ème }}$ siècle. La corbeille de Nefta est réputée pour son point de vue panoramique qui surplombe l'oasis. Le centre de la ville historique, située sur la crête d'une colline, présente les mêmes aspects architecturaux que Tozeur.

6. En 2007, I'Institut National du Patrimoine a organisé les premières rencontres internationales du patrimoine culturel immatériel à l'issue desquelles la Tunisie a ratifié, le 15 février 2007, la Convention sur la protection et la promotion de la diversité des expressions culturelles de 2005.

7. Ashley, Boyd et Goodwin, 2000.

8. Le Décret du 13 Mars 1991

9. II s'agit du projet « Nos ressources, clé de nos emplois " élaboré par l'Association de Sauvegarde de la Médina de Tozeur et financé par l'Agence allemande de coopération internationale (GIZ). Ce projet fait partie d'un plan de restructuration de la corporation et de consolidation de l'activité touristique régionale.
10. Définition adoptée par le Comité Français du Groupe de travail International sur le Tourisme Durable, le 4 juillet 2006.

11. Projet inauguré récemment et fruit d'une collaboration entre le ministère de l'agriculture et des ressources hydrauliques et la Principauté de Monaco.

12. II met en relation des producteurs (trices) agricoles avec des touristes ou des excursionnistes.

13. Le décret stipule qu' « aucun édifice, aucune résidence, aucune construction ne se fera dorénavant sans le recours dans une proportion d'au moins $30 \%$ à la brique locale ».

14. Tozeur compte 50 fabriques de briques traditionnelles.

15. Voir document WHC-12/36.COM/5E, Point 5E de l'ordre du jour provisoire. Programme sur le patrimoine mondial et le tourisme, documents préparatoires à la 36e session du Comité du patrimoine mondial, Saint-Pétersbourg, Fédération de Russie, 24 juin6 juillet 2012.

\section{BIBLIOGRAPHIE}

Abichou, H., Jouve, A-M. et Sghaier, M

(2013). Le tourisme saharien au sud-est tunisien, menace ou vecteur de développement durable des zones marginales. Revue des Régions Arides, 31, pp. 267-275.

Ashley, C., Boyd C. et Goodwin H. (2000): Pro-poor tourism: putting poverty at the heart of the tourism agenda. Natural Resource Perspectives, 51, pp. 1-6.

Boukherouk, M. (2012). La mise en tourisme d'un espace oasien fragile et marginalisé, la région du Tafilalet dans le Sud-est marocain : Enjeux, freins et perspectives [Thèse de doctorat inédite] Université Blaise Pascal : Clermont Ferrand.

Bourdeau, L., Gravari-Barbas, M. et Robinson, M. (dirs.) (2012). Tourisme et Patrimoine Mondial. Québec: Presses de I'Université Laval.

Carpenter, I. et Gana, A. (2012). Les oasis du sud Tunisien, le patrimoine comme le-

vier du développement territorial ? Revue des Régions Arides, 28. pp. 225-237.

Collectif (2006). Expansion du tourisme : gagnants et perdants. Bruxelles: Syllepse.

Cuvelier, P. (1998). Anciennes et nouvelles formes de tourisme, une approche socio-économique. Paris: Le Harmattan.

Daghari, M-H. (2002). Tunisie, habiter sa différence : le bâti traditionnel du SudEst tunisien. Paris: Le Harmattan.

Dhaher, N. (2012a). Espaces traditionnels et fonctions contemporaines : le cas des quartiers historiques de la ville de Tozeur en Tunisie. En Berthold, E. (dir). Les quartiers historiques : pressions, enjeux, actions. Québec: Presses de l'Université Laval, pp. 149-168.

Dhaher, N. (2012b). Les ambivalences de la mise en tourisme du patrimoine. Le cas du centre ancien de Tozeur en Tunisie. Mondes du Tourisme, 6, pp. 23-33. https://doi.org/10.4000/tourisme.232
Dhaher, N. (2016). Mise en tourisme du patrimoine saharien Tunisien ; construction problématique d'une dynamique de développement. Ve colloque sino-européen du tourisme "Tourisme, patrimoine et mémoires au XXIe siècle ». Université de Ningbo.

El Gaied, M. et Meyer, V. (2014). Communication, tourisme et développement territorial : l'exemple des GSOURS du sudest tunisien. Les Enjeux de l'information et de la communication, 15/1, pp. 5-15.

Ezzedine, H. (2000). Le tourisme saharien en Tunisie. Dans Stratégie pour un développement durable du turisme au Sahara. Paris: Unesco, p. 2834. Sur http://unesdoc.unesco.org/ images/0011/001196/119687fo.pdf

Furt, J-M. et Michel, F. (2006). Tourismes et identités. Paris: Le Harmattan.

Garat, I., Gravari-Barbas, M. et Veschambre, V. (2008). Préservation du patrimoine bâti et développement durable : une tautolo- 
gie ? Les cas de Nantes et Angers, Revue Développement durable et territoires. Dossier 4/2008. Sur : https://developpementdurable.revues.org/4913\#text

Gravari-Barbas, M. et S. Jacquot (2013). Patrimoine mondial, Tourisme et développement durable en Afrique. Via@Revue internationale interdisciplinaire de tourisme. Sur : https://viatourismreview. com/fr/2015/06/editorial-world-heritage-tourism-and-sustainable-development-in-africa-discourses-approaches-and-challenges-2/

Magalie, M. et Houda, N. (2004). Économie du Tourisme : Quels acteurs ? Pour quel développement ? Le cas tunisien à la lumière de l'exemple hongrois. Mondes en développement, 125, pp. 33-49. https://doi.org/10.3917/med.125.0033

Minvielle, J-P., Smida, M. et Majdoub, W. (eds.) (2007). Actes du colloque international : tourisme saharien et développement durable : enjeux et approches comparatives : Tozeur (Tunisie). Saint-Quentin-en-Yvelines : Université de Sousse. Sur http:// horizon.documentation.ird.fr/exl-doc/ pleins_textes/divers09-09/010045751.pdf
Neyret, R. et Colin, M. (2003). Lyon, ville ouverte. Les Cuisinieres-Sobbollire.

Office National du Tourisme Tunisien (2015). Le tourisme tunisien en chiffres. Tunis: édition ONTT.

Office National du Tourisme Tunisien (2010). Etude de la gestion durable des systèmes oasiens. Rapport général. Tunis: Ministère de l'environnnement et de développement durable.

Puig, N. (2004). L'opposition bédouins et oasiens, une figure revisitée de l'altérité dans le Sud tunisien. Figures Sahariennes, 7, pp. 1-16.

Sghaïer, M. (2010). Etude de la gouvernance des ressources naturelles dans les oasis. Cas des oasis en Tunisie. [Sur internet]. https://www.iucn.org/sites/ dev/files/import/downloads/etude gouvernance_oasis_tz_final.pdf.

Souissi, M. (2011). Le tourisme de circuit dans le Sahara tunisien : réalités et perspectives. Insaniyat, 51-52, pp. 271-287. https://doi.org/10.4000/insaniyat.12811

\section{Otros recursos}

Babnet Tunisie [Sur internet] http://www. babnet.net/index.php

Écotourisme et tourisme culturel durable en Tunisie. Situation actuelle et perspectives. Rapport du Programme des Nations Unies pour le Développement, 2004. Sur http:// www.abhatoo.net.ma/maalama-textuelle/ developpement-economique-et-social/ developpement-economique/tourisme/ tourisme-ecologique/ecotourisme-et-tourisme-culturel-durable-en-tunisie

Ministère de l'environnnement et de développement durable. Direction génerale de l'environnement et de la qualité de la vie. Stratégie de Développement Durable des Oasis en Tunisie, 2015. Sur http://www. environnement.gov.tn/fileadmin/medias/ pdfs/projet_etude/projet_GDEO/3_1.pdf

Office du développement du sud (ODS). Programme d'amélioration de l'irrigation dans les Oasis du Sud Tunisie, 2012.

Programme des Nations Unies pour l'Environnement (PNUE) et l'Organisation Mondiale du Tourisme (OMT). Vers un Tourisme Durable : Guide à l'usage des décideurs, 2006. 